\title{
Detecção de pré-diabetes e diabetes mellitus em pacientes sem diagnóstico prévio, internados em hospital escola no sul do Brasil
}

\author{
Incidence of undiagnosed prediabetes and diabetes mellitus in admitted \\ patients at the medical clinical ward of a public hospital from Southern \\ Brazil
}

\section{Cristhyan Gabriel Pavlak Polli ${ }^{1}$, Gianna Carla Alberti Schrut ${ }^{1}$, Matheo Augusto Morandi Stumpf ${ }^{1}$, Ana Cláudia Garabeli Cavalli Kluthcovsky ${ }^{1}$}

${ }^{1}$ Universidade Estadual de Ponta Grossa (UEPG), Ponta Grossa, Paraná - Brasil.

\section{Resumo}

Introdução: O diabetes mellitus é uma doença extremamente comum na prática clínica. Ainda não há recomendação de investigação de rotina em pacientes internados e nem qual o teste laboratorial a ser utilizado nesses casos. Objetivo: Detectar pré-diabetes e diabetes mellitus em pacientes sem diagnóstico prévio, internados na ala de clínica médica em hospital universitário no Sul do Brasil e possíveis fatores associados. Metodologia: Estudo observacional, transversal e com abordagem quantitativa. Foram coletados dados sociodemográficos e clínicos. Os valores de hemoglobina glicada foram utilizados para detectar pré-diabetes e diabetes. As comparações entre os grupos foram realizadas pelos testes Quiquadrado de Pearson e Exato de Fisher e as correlações pelo coeficiente correlação de Pearson e Spearman, com nível de significância de 5\%. Resultados: Os 99 pacientes pesquisados tinham idade média de $61,02 \pm 16,93$ anos, $50,51 \%$ do sexo feminino. Foram detectados $27,7 \%$ de pré-diabetes e $3,03 \%$ de diabetes mellitus. Pacientes com asma e/ou DPOC (OR=3,2; IC95\% 1,2-8,4) e glicemia capilar maior de $125 \mathrm{mg} / \mathrm{dL}$ $(\mathrm{OR}=20,7$; IC95\% 6,8-62,4) apresentaram significativamente mais chances de detecção de pré-diabetes ou diabetes mellitus. Houve correlação positiva e significativa entre níveis de hemoglobina glicada com idade e glicemia capilar e entre níveis de pressão arterial sistólica com Índice de Massa Corporal. Conclusão: Observou-se alto percentual de detecção de pré-diabetes, o que sugere a investigação em pacientes hospitalizados por outras causas, especialmente aqueles com diagnóstico de asma e/ou DPOC e com glicemia capilar maior de $125 \mathrm{mg} / \mathrm{dL}$.

Palavras-chave: Diabetes mellitus; diagnóstico; hemoglobina glicada; hospitalização.

\section{Autora correspondente:}




\section{Abstract}

Introduction: Diabetes mellitus is an extremely common disease in clinical practice. There is still no recommendation for routine investigation in hospitalized patients and nor is the laboratory test to be used in these cases. Objective: To detect pre-diabetes and diabetes mellitus in patients without previous diagnosis, hospitalized in the ward of medical clinic in a university hospital in the South of Brazil and possible associated factors. Methodology: Cross-sectional observational study with a quantitative approach. Sociodemographic and clinical data were collected. Glycated hemoglobin values were used to detect pre-diabetes and diabetes. Pearson's Chi-square test and Fisher's exact test were used to compare the groups and the Pearson and Spearman coefficients were used for correlation, with a significance level of 5\%. Results: The 99 patients studied had a mean age of 61,02 \pm 16.93 years, of which 50,51\% were female. Pre-diabetes were detected in 27,7\% and diabetes in 3,03\%. Patients with asthma and/or COPD $(O R=3,2 ;$ CI95\% 1,2-8,4) and capillary glycemia greater than $125 \mathrm{mg} / \mathrm{dL}(\mathrm{OR}=20,7 ; \mathrm{CI} 95 \%$ 6,8-62,4) were significantly more likely to detect pre-diabetes or diabetes mellitus. There was a positive and significant correlation between levels of glycated hemoglobin with age and capillary glycemia, and between levels of systolic blood pressure with Body Mass Index. Conclusion: A high percentage of pre-diabetes detection was observed, which suggests the investigation of patients hospitalized for other causes, especially those diagnosed with asthma and/or COPD and with capillary glycemia greater than $125 \mathrm{mg} / \mathrm{dL}$.

Keywords: Diabetes mellitus; Diagnosis; Glycated hemoglobin; Hospitalization.

\section{Introdução}

O diabetes mellitus (DM) é uma séria ameaça à saúde do mundo todo e que não respeita o status socioeconômico nem as fronteiras nacionais. A Federação Internacional de Diabetes estimou a prevalência mundial da doença em 463 milhões de adultos vivendo com diabetes, e calcula-se que $9,3 \%$ dos adultos entre 20 a 79 anos tenham diabetes. Caso as medidas não sejam suficientes para conter a doença, a projeção é de 700 milhões de pessoas com diabetes no ano de 2045. O Brasil ocupa a quinta posição mundial entre os países com maior número de pessoas com diabetes, apresentando prevalência, em 2019, de 16,8 milhões de pessoas entre 20 e 79 anos, com projeção para 21,5 milhões em 2030. Entre todas as causas de óbito, o diabetes está associado a 11,3\% das mortes entre 20 e 79 anos, equivalente a 1 óbito a cada sete segundos ${ }^{(1)}$.

O diabetes mellitus (DM) é caracterizado por níveis elevados de glicose no sangue de uma pessoa, pela não produção ou produção insuficiente do hormônio insulina, ou por não poder utilizar efetivamente a insulina produzida $^{(1)}$. Um dos exames utilizados para diagnóstico de DM é a determinação dos níveis séricos de hemoglobina glicada, que corresponde à fração da hemoglobina que está ligada à glicose por meio de uma ligação estável e irreversível. Oferece as vantagens por refletir níveis glicêmicos dos últimos 3 a 4 meses, que é o tempo médio de vida das hemácias. Apresentar menor variabilidade dia a dia e independer do estado de jejum para sua determinação ${ }^{(2)}$.

A American Diabetes Association (ADA) ${ }^{(3)}$ e a Sociedade Brasileira de Diabetes ${ }^{(2)}$, estabelecem um dos seguintes critérios para confirmar diagnóstico de DM tipo 2: glicemia de jejum maior ou igual a $126 \mathrm{mg} / \mathrm{dL}$; teste oral com sobrecarga de $75 \mathrm{~g}$ de glicose (TTOG) maior ou igual a $200 \mathrm{mg} / \mathrm{dL}$, após 2h; hemoglobina glicada maior ou igual a 6,5\% ou glicemia aleatória maior ou igual a 200 $\mathrm{mg} / \mathrm{dL}$ na presença de sintomas inequívocos de hiperglicemia. Para diagnóstico de pré-diabetes (pré-DM) é necessário um dos seguintes critérios: glicemia de jejum maior ou igual a 100 e menor que $126 \mathrm{mg} / \mathrm{dL}$, TTGO menor ou igual a 140 e menor que $200 \mathrm{mg} / \mathrm{dL}$ ou hemoglobina glicada maior ou igual a 5,7 e menor que 
6,5\%. A hemoglobina glicada é a medida mais confiável de glicemia em longo prazo $^{(4)}$.

A hiperglicemia de estresse em doenças críticas tem sido associada ao aumento da mortalidade e morbidade em pacientes clínicos e cirúrgicos ${ }^{(5)}$. Pacientes com diabetes mellitus hospitalizados por outros problemas de saúde podem ter maior risco de óbito hospitalar e maior tempo de internação ${ }^{(6)}$. O aumento da mortalidade a curto prazo pode ocorrer em pacientes com hipoglicemia e hiperglicemia na admissão hospitalar, em pacientes com e sem diabetes prévio, independentemente do tratamento, especialidade médica, diagnóstico de alta principal ou valores laboratoriais concomitantes ${ }^{(7)}$. Além disso, em pacientes hospitalizados por doenças infecciosas, o DM está associado a um risco aumentado de mortalidade em longo prazo, especificamente após a hospitalização por infecções respiratórias e de pele e tecidos moles ${ }^{(8)}$.

Estima-se que, para cada dois adultos (20 a 79 anos) com diabetes, há um não diagnosticado. Considerando a subnotificação da doença ${ }^{(1)}$, o momento da hospitalização pode ser uma importante oportunidade para diagnosticar, orientar e começar a tratar casos de diabetes ainda não diagnosticados ${ }^{(9)}$. Devido a subutilização da hemoglobina glicada na avaliação da hiperglicemia em diabéticos não diagnosticado em ambiente hospitalar, uma oportunidade de detecção precoce de DM pode ser perdida ${ }^{(10)}$. O objetivo deste estudo foi detectar pré-DM e DM em pacientes sem diagnóstico prévio, internados na ala de clínica médica em hospital universitário no Sul do Brasil e possíveis fatores associados.

\section{Metodologia}

Trata-se de um estudo observacional, transversal e de abordagem quantitativa, realizado com uma amostra de conveniência de pacientes internados na clínica médica de um hospital universitário no Sul do Brasil.

O período de coleta de dados se deu entre agosto de 2017 a abril de 2018. Os critérios de inclusão foram: ter 18 anos de idade ou mais, estar internado há pelo menos 3 dias, não ter diagnóstico prévio de pré-DM ou DM e aceite em participar da pesquisa. Foi optado por um mínimo de 3 dias de internação para viabilizar a coleta e ter disponível o resultado dos exames laboratoriais. Os critérios de exclusão foram: incapacidade de comunicação/compreensão e estar em uso de corticosteroides por mais de 3 meses. De 213 pacientes internados na ala de clínica médica do hospital, 99 contemplavam os critérios de inclusão e aceitaram participar do estudo.

Caso o paciente fosse incluído no estudo, procedia-se à coleta dos dados sociodemográficos, clínicos e a solicitação da glicemia capilar e hemoglobina glicada. Esse último exame seguiu as recomendações atuais da Sociedade Brasileira de Diabetes e foi realizado por uma das técnicas validadas, a High Performance Liquide Chromatography (HPLC). Os valores de corte da hemoglobina glicada adotados neste estudo foram os publicados pela Sociedade Brasileira de Diabetes, ou seja, maior ou igual a 5,7 e menor que 6,5 para detecção de pré$\mathrm{DM}$ e maior ou igual a $6,5 \%$ para $\mathrm{DM}^{(2)}$. A partir desses resultados, os pacientes foram classificados em dois grupos: aqueles detectados com pré-DM ou DM e aqueles não detectados. Optou-se por utilizar a hemoglobina glicada como padrão-ouro, pois já foi demonstrado que não há alterações significativas deste exame em pacientes internados no início de doença crítica ${ }^{(11)}$.

Os dados sociodemográficos e clínicos analisados foram: sexo, idade, tabagismo atual, doenças atuais (hipertensão arterial sistêmica, dislipidemia, cardiopatia e asma/doença pulmonar obstrutiva crônicaDPOC), índice de massa corporal (IMC $\geq 30 \mathrm{~kg} / \mathrm{m}^{2}$ obesidade e $<30 \mathrm{~kg} / \mathrm{m}^{2}$ não obesidade) ${ }^{(2)}$, medicamentos utilizados atualmente, pressão arterial sistólica (PAS $\geq 140$ mmHg não controlada e $<140 \mathrm{mmHg}$ controlada $)^{(2)}$, pressão arterial diastólica (PAD $\geq 90 \quad \mathrm{mmHg}$ não controlada e $<90 \mathrm{mmHg}$ controlada) ${ }^{(2)}$, média do número de dias de internamento até a coleta dos exames $(\geq 5 \mathrm{e}<5)$, internamento na Unidade de Terapia Intensiva (UTI), evolução para óbito durante o internamento e glicemia sanguínea aleatória $(>125 \mathrm{mg} / \mathrm{dL}$ alterado e $\leq 125 \mathrm{mg} / \mathrm{dL}$ 
não alterado $)^{(9)}$. Neste estudo foi utilizada glicemia capilar aleatória.

A descrição das variáveis foi realizada pela estatística descritiva. As variáveis quantitativas foram relatadas pelos valores da média e desvio-padrão e as variáveis qualitativas por frequências absolutas e relativas. Foram utilizados histogramas e teste de ShapiroWilk para verificação de gaussianidade. As comparações entre os grupos detectados ou não com pré-DM e DM foram realizadas pelos testes Qui-quadrado de Pearson e Exato de Fisher. As correlações entre as variáveis idade e clínicas com a hemoglobina glicada e níveis de PAS e PAD foram realizadas pelos coeficientes de Correlação de Pearson e Spearman.

Os dados foram processados no banco de dados Excel 2010. A análise estatística foi obtida com auxílio do programa Statistical Package for Social Science (SPSS), versão 15.0 e o nível de significância utilizado foi de $5 \%$.

O projeto foi aprovado pelo Comitê de Ética em Pesquisa (CAAE: 76710017.2.0000.0105, parecer CEP 2.305.869) e, antes da coleta de dados, todos os pacientes participantes assinaram o Termo de Consentimento Livre e Esclarecido, seguindo todos os preceitos éticos para pesquisa envolvendo seres humanos.

\section{Resultados}

Dentre os 99 pacientes pesquisados, 49 eram do sexo masculino $(49,49 \%)$ e 50 do sexo feminino (50,51\%), com média de idade de 61,02 $\pm 16,93$ anos. Os valores de hemoglobina glicada variaram de 4,28 a 6,98\%,

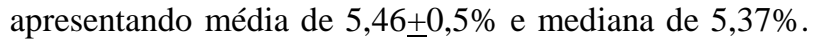
Ao classificar os pacientes de acordo com os critérios da Sociedade Brasileira de Diabetes ${ }^{(2)}$, encontramos: 3 pacientes $(3,03 \%)$ com DM e $27(27,7 \%)$ com pré-DM.

A TABELA 1 apresenta a distribuição dos grupos detectados ou não com pré-DM e DM, segundo variáveis sociodemográficas e clínicas. Das variáveis analisadas, ter diagnóstico de asma e/ou DPOC representou uma chance 3,2 vezes maior para detecção de pré-DM ou DM (OR=3,2; IC95\% 1,2-8,4). Outra variável significativa foi a glicemia capilar, uma vez que valor maior do que $125 \mathrm{mg} / \mathrm{dL}$ representou uma chance 20,7 vezes maior para detecção de pré-DM ou DM $(\mathrm{OR}=20,7$; IC 95\% 6,8-62,4). Apesar do sexo masculino ter representado $63,3 \%$ e a HAS $73,3 \%$ dentre os pacientes detectados com pré-DM ou DM, essas duas variáveis não apresentaram significância estatística na comparação com os pacientes não detectados, assim como as demais variáveis. 
TABELA 1 - Distribuição dos pacientes detectados com pré-diabetes ou diabetes mellitus, segundo variáveis sociodemográficas e clínicas $(\mathbf{n}=99)$

\begin{tabular}{|c|c|c|c|c|}
\hline \multirow{2}{*}{ Variáveis } & \multicolumn{2}{|c|}{$\begin{array}{c}\text { Detecção de pré-diabetes ou } \\
\text { diabetes mellitus }\end{array}$} & \multirow[b]{2}{*}{$p$} & \multirow{2}{*}{ OR (IC95\%) } \\
\hline & $\begin{array}{c}\operatorname{Sim}(\mathbf{n}=30) \\
\mathbf{n}(\%)\end{array}$ & $\begin{array}{l}\text { Não(n=69) } \\
\text { n }(\%)\end{array}$ & & \\
\hline Sexo & & & $0,07 *$ & $2,2(0,9-5,4)$ \\
\hline Masculino & $19(63,3)$ & $30(43,5)$ & & \\
\hline Feminino & $11(36,7)$ & $39(56,5)$ & & \\
\hline Idade em anos & & & $0,18 *$ & $1,8(0,7-4,5)$ \\
\hline$<60$ & $20(66,7)$ & $36(52,2)$ & & \\
\hline$\geq 60$ & $10(33,3)$ & $33(47,8)$ & & \\
\hline Tabagismo & & & $0,62 *$ & $1,3(0,4-4,0)$ \\
\hline $\operatorname{Sim}$ & $6(20,0)$ & $11(15,9)$ & & \\
\hline Não & $24(80,0)$ & $58(84,1)$ & & \\
\hline HAS & & & $0,35 *$ & $1,6(0,6-4,0)$ \\
\hline Sim & $22(73,3)$ & $44(63,8)$ & & \\
\hline Não & $8(26,7)$ & $25(36,2)$ & & \\
\hline Dislipidemia & & & $0,16^{*}$ & $2,0(0,7-5,5)$ \\
\hline $\operatorname{Sim}$ & $9(30,0)$ & $12(17,4)$ & & \\
\hline Não & $21(70,0)$ & $57(82,6)$ & & \\
\hline Cardiopatia & & & $0,17 *$ & $1,9(0,7-4,9)$ \\
\hline Sim & $11(36,7)$ & $16(23,2)$ & & \\
\hline Não & $19(63,3)$ & $53(76,8)$ & & \\
\hline Asma/DPOC & & & $0,01 *$ & $3,2(1,2-8,4)$ \\
\hline $\operatorname{Sim}$ & $13(43,3)$ & $13(18,8)$ & & \\
\hline Não & $17(56,7)$ & $56(81,2)$ & & \\
\hline Obesidade & & & $0,67 * *$ & $0,7(0,2-3,0)$ \\
\hline $\mathrm{IMC} \geq 30$ & $3(10,0)$ & $9(13,0)$ & & \\
\hline $\mathrm{IMC}<30$ & $27(90,0)$ & $60(87,0)$ & & \\
\hline Medicamentos em uso & & & $0,26 *$ & $1,6(0,6-3,9)$ \\
\hline$\leq 3$ & $16(53,3)$ & $45(65,2)$ & & \\
\hline$>3$ & $14(46,7)$ & $24(34,8)$ & & \\
\hline Pressão arterial sistólica & & & $0,36 *$ & $1,5(0,6-3,8)$ \\
\hline$\geq 140$ & $11(36,7)$ & $19(27,5)$ & & \\
\hline$<140$ & $19(63,3)$ & $50(72,5)$ & & \\
\hline $\begin{array}{l}\text { Pressão arterial } \\
\text { diastólica }\end{array}$ & & & $0,47 *$ & $1,4(0,5-3,7)$ \\
\hline$\geq 90$ & $9(30,0)$ & $16(23,2)$ & & \\
\hline$<90$ & $21(70,0)$ & $53(76,8)$ & & \\
\hline $\begin{array}{l}\text { Dias de internamento } \\
\text { até a coleta dos exames }\end{array}$ & & & $0,35^{*}$ & $0,6(0,2-1,6)$ \\
\hline$\geq 5$ & $8(26,7)$ & $25(36,2)$ & & \\
\hline$<5$ & $22(73,3)$ & $44(63,8)$ & & \\
\hline UTI & & & $0,27 *$ & $1,6(0,6-3,9)$ \\
\hline Sim & $13(43,3)$ & $22(31,9)$ & & \\
\hline Não & $17(56,7)$ & $47(68,1)$ & & \\
\hline Óbito no internamento & & & $0,51 * *$ & - \\
\hline Sim & $0(0,0)$ & $1(1,4)$ & & \\
\hline Não & $30(100,0)$ & $68(98,6)$ & & \\
\hline Glicemia capilar & & & $<0,001 *$ & $20,7(6,8-62,4)$ \\
\hline$>125$ & $21(70,0)$ & $7(10,1)$ & & \\
\hline$\leq 125$ & $9(30,0)$ & $62(89,9)$ & & \\
\hline
\end{tabular}

DPOC=doença pulmonar obstrutiva crônica. *teste Qui-quadrado de Pearson. **Teste Exato de Fisher. Os valores totais apresentam pequena variação devido a algumas informações faltantes para a variável.

A TABELA 2 apresenta as correlações entre idade e variáveis clínicas com a hemoglobina glicada,
PAS e PAD. Houve correlação positiva e significativa entre os níveis de hemoglobina glicada e idade ( $\mathrm{r}=0,29$; 
$\mathrm{p}<0,01)$ e glicemia capilar $(\mathrm{r}=0,55 ; \mathrm{p}<0,001)$. Também se observou correlação positiva e significativa entre PAS e

$\operatorname{IMC}(r=0,24 ; p=0,02)$.

TABELA 2 - Correlação da idade e variáveis clínicas com a hemoglobina glicada, pressão arterial sistólica e diastólica $(\mathbf{n}=99)$

\begin{tabular}{|c|c|c|c|c|c|c|}
\hline \multirow{2}{*}{ Variáveis clínicas } & \multicolumn{2}{|c|}{$\begin{array}{c}\text { Hemoglobina } \\
\text { glicada }\end{array}$} & \multicolumn{2}{|c|}{$\begin{array}{c}\text { Pressão arterial } \\
\text { sistólica }\end{array}$} & \multicolumn{2}{|c|}{$\begin{array}{c}\text { Pressão arterial } \\
\text { diastólica }\end{array}$} \\
\hline & $\mathrm{r}$ & $p$ & $\mathrm{r}$ & $p$ & $\mathrm{r}$ & $p$ \\
\hline Idade & 0,29 & $<\mathbf{0 , 0 1} * *$ & 0,00 & $100 * *$ & $-0,19$ & $0,06 * *$ \\
\hline IMC & 0,10 & $0,35 *$ & 0,24 & $\mathbf{0 , 0 2} *$ & 0,07 & $0,50 * *$ \\
\hline Glicemia capilar & 0,55 & $<0,001 *$ & 0,11 & $0,27 *$ & $-0,00$ & $1,00 * *$ \\
\hline Total de dias de internação & $-0,04$ & $0,71 * *$ & 0,00 & $0,98 * *$ & $-0,03$ & $0,75^{* *}$ \\
\hline Ureia & 0,17 & $0,10 * *$ & 0,19 & $0,06^{* *}$ & 0,09 & $0,39 * *$ \\
\hline Creatinina & 0,03 & $0,77 * *$ & 0,12 & $0,26^{* *}$ & $-0,06$ & $0,56 * *$ \\
\hline Hemoglobina & 0,14 & $0,16^{*}$ & 0,19 & $0,07 *$ & $-0,06$ & $0,56^{* *}$ \\
\hline Leucócitos & 0,07 & $0,47 * *$ & 0,12 & $0,29 * *$ & 0,01 & $0,92 * *$ \\
\hline Plaquetas & 0,07 & $0,47 * *$ & 0,05 & $0,63 * *$ & 0,07 & $0,48 * *$ \\
\hline Sódio & $-0,02$ & $0,85^{*}$ & 0,16 & $0,12 *$ & 0,07 & $0,49 * *$ \\
\hline Potássio & 0,12 & $0,25 *$ & 0,03 & $0,77^{*}$ & $-0,08$ & $0,46^{* *}$ \\
\hline
\end{tabular}

*Coeficiente de Correlação de Pearson **Coeficiente de Correlação de Spearman IMC = Índice de Massa Corporal $\mathrm{r}=$ valor da correlação

\section{Discussão}

Neste estudo, foi possível confirmar a existência de subdiagnóstico de DM e pré-DM em uma população de pacientes internados, e foram encontrados valores relativamente elevados. Aproximadamente $30 \%$ da amostra não sabia que era portadora de alguma dessas condições. Maior acesso da população ao diagnóstico precoce do diabetes seria de muita importância, já que, globalmente, estima-se que $45,8 \%$, ou 174,8 milhões de todos os casos de diabetes em adultos são não diagnosticados, e $83,8 \%$ de todos os casos não diagnosticados ocorram em países de baixa e média renda (12).

Alguns estudos apontam a presença de DM sem diagnóstico prévio em algumas populações estudadas. Ekinci e colaboradores ${ }^{(13)}$ analisaram uma amostra de 416 pacientes com idade maior ou igual a 54 anos que estavam internados em uma unidade ortopédica. Do total, $22 \%$ dos pacientes sabiam ser diabéticos, $4 \%$ tinham diabetes não reconhecido anteriormente e $74 \%$ não tinham diabetes. Após ajuste para idade, sexo, escore de comorbidade e taxa de filtração glomerular estimada, não houve diferenças significativas no tempo de internação, taxas de internação em UTI, mortalidade em 6 meses, readmissão hospitalar em 6 meses, ou quaisquer complicações pósoperatórias entre os pacientes com e sem diabetes.

Em uma coorte prospectiva australiana em 5.082 com 54 anos ou mais, foi encontrado $5 \%$ de prevalência de DM não previamente diagnosticado. $\mathrm{O}$ ponto de corte utilizado para diagnóstico de DM também foi o valor maior ou igual a $6,5 \%$ para hemoglobina glicada. Depois de ajustar para idade, sexo, pontuação do Índice de Charlson, taxa de filtração glomerular estimada e níveis de hemoglobina, com unidade de internação tratada como um efeito aleatório, os pacientes com diabetes previamente conhecido tiveram mortalidade menor em 6 meses. Não houve diferença em relação à admissão na UTI, uso de ventilação mecânica ou readmissão hospitalar, quando comparados os grupos de pacientes ${ }^{(14)}$.

Foi observado que pacientes com diagnóstico de asma e/ou DPOC apresentaram maiores chances de detecção de pré-DM ou DM no presente estudo. De fato, há estudos que sugerem associação entre DM2 e a DPOC (15,16). Uma revisão de literatura encontrou evidências da associação entre DM2 com DPOC, com diversos fatores envolvidos, como inflação, estresse oxidativo, disfunção 
muscular esquelética, infecções pulmonares, alterações no metabolismo das adipocinas e uso de corticosteroides. $\mathrm{O}$ diabetes pode atuar como um fator independente, afetando negativamente a estrutura e a função $\operatorname{pulmonar}^{(17)}$. No Reino Unido, Feary e colaboradores ${ }^{(18)}$ analisaram 1.204.100 prontuários e encontraram um pequeno aumento nas chances de pacientes com DPOC apresentarem $\mathrm{DM} \quad(\mathrm{OR}=2,04 ; \mathrm{IC} 95 \%$ 1,97-2,12), semelhante ao encontrado neste estudo.

Outra variável analisada que demonstrou maior chance de detecção de pré-DM ou DM foi a glicemia capilar aleatória maior do que $125 \mathrm{mg} / \mathrm{dL}(\mathrm{OR}=20,7$; IC95\% 6,8-62,4). Além disso, observou-se correlação positiva e significativa entre os níveis de glicemia capilar e hemoglobina glicada para todos os pacientes pesquisados. Greci e colaboradores ${ }^{(9)}$ propuseram um algoritmo para solicitação de hemoglobina glicada em 508 pacientes admitidos no departamento de emergência de um hospital. Em seu estudo, pacientes com glicemia plasmática aleatória acima de $125 \mathrm{mg} / \mathrm{dL}$ se beneficiaram da solicitação da hemoglobina glicada como um teste de triagem inicial para o diagnóstico de DM.

Neste estudo, observou-se correlação significativa e positiva entre idade e hemoglobina glicada. Possivelmente este achado seja devido ao avançar da idade, já que a prevalência da doença é maior entre as pessoas $\operatorname{idosas}^{(1)}$. Além disso, parece haver maior prevalência de DM em pacientes internados e com maior idade $^{(19)}$. Também foi encontrada correlação positiva e significativa entre níveis de PAS e IMC. De fato, correlação positiva entre obesidade e hipertensão arterial sistêmica está descrita na literatura ${ }^{(20)}$.

Sabe-se que com um diagnóstico rápido e preciso e cuidados adequados, o diabetes é uma doença que pode ser controlada e suas complicações evitadas. Além disso, muitas vezes, o DM2 pode ser prevenido, uma vez que há evidências as quais sugerem a possibilidade de reversão, em algumas circunstâncias. Assim, os governos e o setor privado devem ser estimulados a tomar medidas para identificar o diabetes não diagnosticado, prevenir o diabetes tipo 2 em pessoas de risco e melhorar a assistência para as pessoas que vivem com a doença ${ }^{(1)}$.
Este estudo apresentou algumas limitações, como a realização em um centro único, um vez que os resultados não podem ser generalizados. Também o pequeno número amostral, em comparação com estudos semelhantes internacionais. Apesar das limitações, o estudo contribuiu para um melhor entendimento sobre a detecção de pré-DM e DM em pacientes sem diagnóstico prévio e hospitalizados por outras causas.

\section{Conclusão}

Observou-se alto percentual de detecção de préDM, o que sugere a investigação em pacientes hospitalizados por outras causas, sem diagnóstico prévio para pré-DM ou DM. Isso pode ser uma alternativa para os pacientes que não tiveram a oportunidade de realizar exames de diagnóstico na atenção primária à saúde, além de possibilitar o início do acompanhamento ou tratamento adequados. Mais estudos sobre o tema poderiam ser realizados, preferencialmente longitudinais e que considerassem o custo-efetividade.

\section{Declaração de Conflito de Interesses}

Os autores do artigo afirmam que não houve qualquer tipo de conflito de interesses que pudesse influenciar no desenvolvimento do trabalho.

\section{Referências}

\section{INTERNATIONAL DIABETES FEDERATION}

(IDF). IDF Diabetes Atlas, 9th edition. Brussels,

Belgium: International Diabetes Federation, 2019.

Disponível em:

https://www.diabetesatlas.org/en/resources/. Aceso em: 10 set. 2020.

2. SOCIEDADE BRASILEIRA DE DIABETES (SBD), Diretrizes Sociedade Brasileira de Diabetes, 20192020. São Paulo (SP); 2019.

\section{AMERICAN DIABETES ASSOCIATION (ADA).}

Standards of Medical Care in Diabetes - 2020. Diabetes

Care, v.43, Supplement 1, p.S14-S31, 2020. 
4. IKEDA, M.; SHIMAZAWA, R. Challenges to hemoglobin A1c as a therapeutic target for type 2 diabetes mellitus. Journal of General and Family Medicine, v.20, n.4, p.129-138, 2019.

5. BAR-OR, D. et al. Stress Hyperglycemia in Critically Ill Patients: Insight Into Possible Molecular Pathways.

Frontiers in Medicine (Lausanne), v.6, n.54, p.1-6, 2019.

6. VALENT, F; TONUTTI, L; GRIMALDI, F. Does diabetes mellitus comorbidity affect in-hospital mortality and length of stay? Analysis of administrative data in an Italian Academic Hospital. Acta Diabetologica, v. 54, n. 12, p. 1081-1090, 2017.

7. BJÖRK, M.; MELIN, E. O.; FRISK, T.;

THUNANDER, M. Admission glucose level was associated with increased short-term mortality and length-of-stay irrespective of diagnosis, treating medical specialty or concomitant laboratory values. European Journal of Internal Medicine, v. 75, p. 71-78, 2020. 8. AKIROV, A.; DIKER-COHEN, T.; MASRI-IRAQI, H.; DUSKIN-BITAN, H.; SHIMON, I.; GORDSHTEIN, A. Outcomes of Hyperglycemia in Patients With and Without Diabetes Mellitus Hospitalized for Infectious Diseases. Diabetes/Metabolism Research and

Reviews, v. 17, e3027, 2018.

9. GRECI, L.S.; KAILASAM, M.; MALKANI, S.; KATZ, D.L.; HULINSKY, I.; AHMADI, R.; NAWAZ, H. Utility of HbA1c Levels for Diabetes Case Finding in Hospitalized Patientes With Hyperglycemia. Diabetes Care, v. 26, n. 4, p. 1064-1068, 2003.

10. JONES, D.; SCHARFENBERG, B.; PERKINS, J.; CHILDERS, K.; DOGBEY. G.Y.; SHUBROOK, J.H. Glycated Hemoglobin Testing to Identify Undiagnosed Diabetes Mellitus in the Inpatient Sentting. Journal of the American Osteopathic Association, v. 116, n. 6, p. 350-357, 2016.

11. LUETHI, N.; , CIOCCARI, L.; TANAKA, A.; KAR, P.; GIERSCH, E.; DEANE, A.M.; MÅRTENSSON, J.; BELLOMO, R. Glycated Hemoglobin A1C Levels Are Not Affected by Critical Ilness. Critical Care Medicine, v. 44, n. 09, p. 1692-1694, 2016.
12. BEAGLEY, J.; GUARIGUATA, L.; WEIL, C.;

MOTALA, A.A. Global estimates of undiagnosed diabetes in adults. Diabetes Research Clinical and Practice, v. 103, n. 2, p. 150-160, 2014.

13. EKINCI, E.I.; KONG, A.; CHURILOV, L.; NANAYAKKARA, N.; CHIU, W.L.; SUMITHRAN, P.; DJUKIADMODJO, F.; PREMARATNE, E.; OWENJONES, E.; HART, G.K.; ROBBINS, R.; HARDIDGE, A.; JOHNSON, D.; BAKER, S.T.; ZAJAC, J.D. Using Automated HbA1c Testing to Detect Diabetes Mellitus in Orthopedic Inpatients and Its Effect on Outcomes. Bencharit S, ed. PLoS ONE, v. 12, n. 1, e0168471, 2017.

14. NANAYAKKARA, N.; NGUYEN, H.; CHURILOV, L.; KONG, A.; PANG, N.; HART, G.K.; OWENJONES, E.; WHITE, J.; ROSS, J.; STEVENSON, V.; BELLOMO, R.; LAM, Q.; CRINIS, N.; ROBBINS, R.; JOHNSON, D.; BAKER, S.T.; ZAJAC, J.D.; EKINCI, E.I. Inpatient HbA1c testing: a prospective observational study. BMJ Open Diabetes Research \& Care, v.3, n. 1, e000113, 2015.

15. PINO-SEDEÑO, T.D. et al. Relationship between glycemic control and chronic obstructive pulmonary disease in patients with type 2 diabetes: A nested casecontrol study. Primary Care Diabetes, v.S1751-9918, n.20, p.30192-3, 2020.

16. GAYLE, A. et al. Incidence of type II diabetes in chronic obstructive pulmonary disease: a nested casecontrol study. NPJ Primary Care Respiratory Medicine, v.29, n.1, p.28, 2019.

17. MIRRAKHIMOV, A.E. Chronic obstructive pulmonary disease and glucose metabolism: a bitter sweet symphony. Cardiovascular Diabetology, v.11, v. $11: 132,2012$.

18. FEARY, J.R.; RODRIGUES, L.C.; SMITH, C.J.; HUBBARD, R.B.; GIBSON, J.E. Prevalence of major comorbidities in subjects with COPD and incidence of myocardial infarction and stroke: a comprehensive analysis using data from primary care. Thorax, v. 65, n. 11, p. 956-962, 2010.

19. MAYEGA, R.W.; RUTEBEMBERWA, E. Clinical presentation of newly diagnosed diabetes patients in a 
rural district hospital in Eastern Uganda. African Health

Science, v.18, n.3, p.707-719, 2018.

20. JIANG, S.Z.; LU W.; ZONG, X.F.; RUAN, H.Y.;

LIU, Y. Obesity and hypertension. Experimental and

Therapeutic Medicine, v. 12, n. 4, p. 2395-2399, 2016. 